\title{
Seismology and activity of the F type star HD $49933^{\star}$
}

\author{
B. Mosser ${ }^{1}$, F. Bouchy ${ }^{2}$, C. Catala ${ }^{1}$, E. Michel ${ }^{1}$, R. Samadi ${ }^{1}$, F. Thévenin ${ }^{3}$, P. Eggenberger ${ }^{4}$, \\ D. Sosnowska ${ }^{4}$, C. Moutou ${ }^{2}$, and A. Baglin ${ }^{1}$ \\ 1 LESIA, CNRS UMR 8109, Observatoire de Paris, 92195 Meudon Cedex, France \\ e-mail: benoit.mosser@obspm.fr \\ 2 LAM, CNRS UMR 6110, Traverse du Siphon, BP 8, 13376 Marseille Cedex 12, France \\ 3 Laboratoire Cassiopée, CNRS UMR 6202, Observatoire de la Côte d'Azur, BP 4229, 06304 Nice Cedex 4, France \\ 4 Observatoire de Genève, 51 ch. des Maillettes, 1290 Sauverny, Switzerland
}

Received 19 October 2004 / Accepted 11 January 2005

\begin{abstract}
A 10-night asteroseismic observation programme has been conducted in January 2004 with the spectrometer HARPS at the ES0 3.6-m telescope. The selected target, the 6th magnitude F5V star HD 49933, was chosen among the prime candidates of COROT, the European space mission dedicated to characterize stellar oscillations mode with high precision photometry measurements. This star shows important line profiles variations, indicating a surprisingly high activity with respect to its low rotation rate. However, with the help of tools developed for disentangling the signatures of activity and oscillations, we are able to observe its oscillation spectrum in the frequency range [1.2, $2.2 \mathrm{mHz}]$. We measure the large separation $(88.7 \pm$ $0.4 \mu \mathrm{Hz}$ ) and the maximum amplitude (around $0.4 \pm 0.1 \mathrm{~m} \mathrm{~s}^{-1} \mathrm{rms}$ ), respectively in agreement and marginal agreement with the predicted values.
\end{abstract}

Key words. techniques: radial velocities - stars: oscillations

\section{Introduction}

F type stars lack seismic observations owing to the difficulty, due to the poor number of their spectral lines, to get a high accuracy seismic signal with ground-based spectrometric measurements. In this study, we present an asteroseismic observational programme conducted with the new generation spectrometer HARPS, installed at the ESO 3.6-m telescope in La Silla (Pepe et al. 2002) on a F type star which is a candidate target (HD 49933) of the space COROT mission (Baglin et al. 2002). The unique capabilities of the spectrograph HARPS made possible to measure, to identify and to characterize the solar-like oscillations of such a 6 th magnitude F star.

In this letter, we present first the observations (Sect. 2), then describe the long term radial velocity pattern we observed (Sect. 3). The method we used in order to correctly extract the radial velocities is exposed in Sect. 4. The seismic signature is confirmed and discussed in Sect. 5. Section 6 is devoted to conclusions.

\footnotetext{
* Based on observations obtained with the HARPS échelle spectrometer mounted on the 3.6-m telescope at ESO-La Silla Observatory (Chile).
}

\section{Observations}

HD 49933 (HR 2530, ADS 5505A) was selected among the COROT prime targets. This F5 V star lies in a region of the HR diagram not yet explored by previous seismic observations. HD 49933 has an effective temperature of $6500 \pm 100 \mathrm{~K}$ with an estimated absolute magnitude $M_{\mathrm{V}}=3.39 \pm 0.10$. This star, with an iron abundance of -0.37 dex (Solano et al. 2004), is sligthly metal poor compared to the Sun and to Procyon. From observations of solar-like oscillations in Doppler velocity, Samadi et al. (2004) find that the maximum in the oscillation velocity, $V_{\max }$, scales approximatively as $(L / M)^{0.65}$ where $L$ and $M$ are the luminosity and the mass of the star respectively. Such a scaling law then predicts $V_{\max } \simeq 60 \pm 0.5 \mathrm{~cm} \mathrm{~s}^{-1} \mathrm{rms}$ for HD 49933 . Spectrometric seismic observations being limited by the stellar line width, we had to select a slow rotator: HD 49933 had an estimated $v \sin i$ about $10.9 \mathrm{~km} \mathrm{~s}^{-1}$ (Solano et al. 2004), changed to $9.5 \pm 0.3 \mathrm{~km} \mathrm{~s}^{-1}$ with our more precise measurements.

About $57 \mathrm{~h}$ observations were recorded between January 15 and 25 , representing 1351 measurements. The exposure time was $120 \mathrm{~s}$, giving one measurement each $153 \mathrm{~s}$ when including overhead, and a Nyquist frequency about $3.3 \mathrm{mHz}$ above the predicted cutoff frequency at $2.2 \mathrm{mHz}$. The mean SNR in the spectrum (at $550 \mathrm{~nm}$ and with a spectral bin of $0.85 \mathrm{~km} \mathrm{~s}^{-1}$ ) typically varies from 60 at high airmass to about 120 . This SNR corresponds exactly to the contribution of the photon 


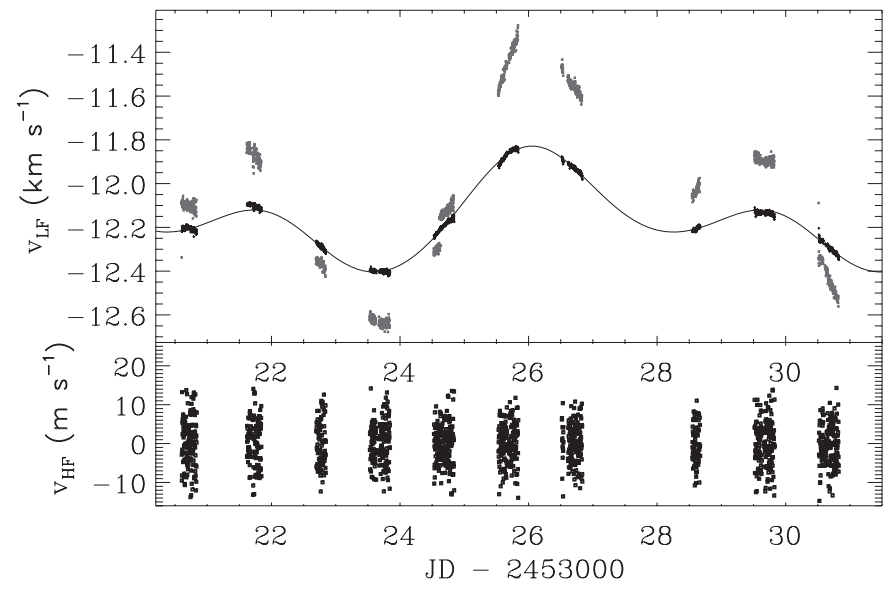

Fig. 1. Time series of the radial velocity of HD 49933 measured with the pipeline reduction of HARPS. The average line bisector amplitudes, multiplied by the sign of the mean slope of the bisectors, are superimposed (in grey) and translated for display purposes. The full signal (upper window) shows large variations, with a period about 7.9 days. The high frequency component (lower window) was corrected from the low frequency fit (thin solid line in the upper window) and also from the residual offsets and slopes.

noise, which varies rapidly with the airmass, as the input flux in the entrance fiber. The mean observation duration did not exceed $7.5 \mathrm{~h}$ per night at this summer period.

\section{Long term radial velocity pattern, line asymmetries and activity}

The radial velocity curve of HD 49933, determined with the online pipeline reduction of HARPS, shows large amplitude variations with a characteristic time scale of a few days (Fig. 1). Further measurements with the CORALIE spectrometer on the Swiss 1.2-m telescope (Queloz et al. 2000) revealed that this long term radial velocity pattern is not stable over periods longer than 10 days, and cannot be attributed to one or several orbiting giant planets.

Suspecting that long term variations may be the signature of stellar activity, we examined the spectral line bisectors in our spectra. We first constructed average photospheric profiles, combining the profiles of the many lines in the HARPS spectra using the Least-Square Deconvolution (LSD) method described in Donati et al. (1997), the line pattern being constructed using a Kurucz model with $T_{\text {eff }}=6500 \mathrm{~K}, \log g=4.5$, and $[\mathrm{Fe} / \mathrm{H}]=-0.3$. These parameters represent the closest choice to those of HD 49933 in the model grids we have at our disposal. We have checked that using adjacent models in the grid does not modify the results. Typical signal-to-noise ratios of 600 to 700 are obtained per velocity bin of $0.2 \mathrm{~km} \mathrm{~s}^{-1}$ in the LSD average profiles. The bisectors of the resulting average line profiles were then constructed as the midpoints of horizontal segments bounded by the sides of the profiles. We find that the line bisectors of HD 49333 most often exhibit the classical C-shape of cool stars, although with a larger distortion in the lower part than in the upper part of the line, in contrast to most other observed cool star bisectors (Gray 1992). They have amplitudes up to about $1 \mathrm{~km} \mathrm{~s}^{-1}$, measured as the

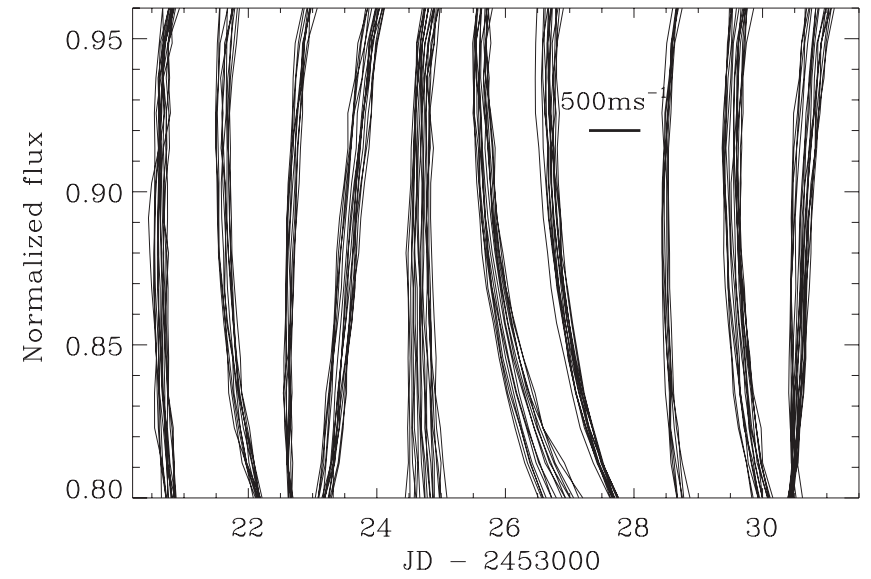

Fig. 2. Average line bisector amplitude of HD 49933. The large variations identified in Fig. 1 are related to the stellar activity modulated by the rotation of the star.

velocity span covered by the bisector, and they are highly variable. The bisector variation is well visible in Fig. 2, with the 8day periodicity suggested by the radial velocity curve in Fig. 1. We conclude that this long-term radial velocity variation can be entirely attributed to line profile distortions, which are most likely related to stellar surface activity. The 8-day periodicity may be identified as rotational modulation of the surface pattern giving rise to the variations. One probable interpretation is that the line distortions are due to the presence of one or several cool spots at the stellar surface, whose signature crosses the line profiles from blue to red during the star rotation, thus affecting the line bisectors. This interpretation is fully compatible with the apparent double-periodicity of the signal. The bumps produced by stellar spots are stronger near the bottom of the lines than in the line wings, which may explain the stronger variations of the line bisectors near their lower end.

Line bisector variations have already been reported in several cases, mostly for $\mathrm{G}$ and $\mathrm{K}$ type stars (López-Santiago et al. 2003 and references therein), the bisector modulation being interpreted as the signature of one or several cool starspots at the stellar surface. The observed bisector modulation amplitudes are usually small. In comparison to these results, the larger amplitude $\left(1 \mathrm{~km} \mathrm{~s}^{-1}\right)$ for the bisector modulation of HD 49933 is somewhat surprising for such a slow rotator ( $v \sin i=9.5 \mathrm{~km} \mathrm{~s}^{-1}, P_{\text {rot }} \simeq 8$ days). The detailed interpretation of these results and the modelling of the stellar surface structures will be proposed in a further paper.

\section{Disentangling Doppler shifts and line profile variations}

The signature of activity occuring at low frequency should not affect the seismic signal at higher frequency. However, the large distortions of the lines related to activity may mimic rapidly varying tiny Doppler shifts. Therefore, any method for extracting the radial velocity must be able to distinguish between a line distortion and a pure line shift. The numerical crosscorrelation technique (Baranne et al. 1996) of the reduction pipeline of HARPS uses as an input the theoretical position of 


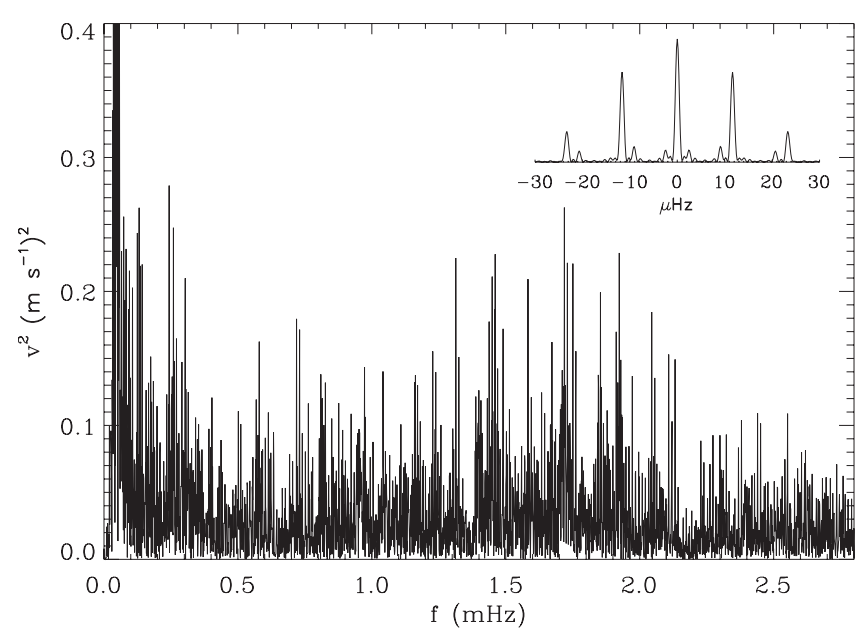

Fig. 3. Fourier spectrum of the velocity time series calculated with the optimum method. The window function is shown in the inset. No high pass filtering was applied, except for the removal of the mean velocity slope of each night.

the stellar lines. The mean line profile is then reconstructed by interpolation of the observed lines corresponding to the theoretical positions. A Gaussian fit gives the Doppler shift, which is affected by the line profile. Therefore, deformations of the line profile may give a spurious Doppler-like signal. The optimum method (Connes 1985; Bouchy et al. 2001) is in principle independent of the line profile. Any spectral line is compared to itself at different times of observations and the wavelength shift is translated into a Doppler shift according to a first order expansion. The efficiency of the method requires a reference spectrum with a high SNR. With the present set of observations, the method proves to be efficient in practive with a reference spectrum having a SNR greater than 500, resulting of the addition of more than 30 spectra. Therefore, the line profiles of the reference and of the spectra do not coincide, so that Doppler artefacts may be induced by lines distortions. Finally, both reduction methods give time series with similar velocity noise of about $3.5 \mathrm{~m} \mathrm{~s}^{-1}$ per integration time (Fig. 1). However, we cannot be sure that the signal in the Fourier spectrum plotted in Fig. 3 is not appreciably contaminated by the stellar activity.

In order to disentangle the distortion and Doppler shift as precisely as possible, we have analysed the LSD profile by decomposing it in two terms. The mean line profile $I(t)$ being considered as a function of the velocity $v$, the intensities $I(t, v)$ and $I(t,-v)$ explore it symmetrically, and give the integrated quantities:

$I_{+,-}(t)=\sum_{0}^{V} w(v)[I(t, v) \pm I(t,-v)]$.

The normalized weighting function $w(v)$ is calculated from a Gaussian fit of $I$, with $w$ proportional to the derivative of this fit, this enhancing the role of the line wings comparatively to the continuum and the line center. The velocity $V$ is high enough for covering the whole profile. The spectrum of the symmetric component $I_{+}(t)$ shows no excess power in the range [1.2, $2.2 \mathrm{mHz}$ ] as seen in Fig. 4, contrary to the antisymmetric signal $I_{-}(t)$, which is very similar to the spectrum of Fig. $3: I_{+}$is

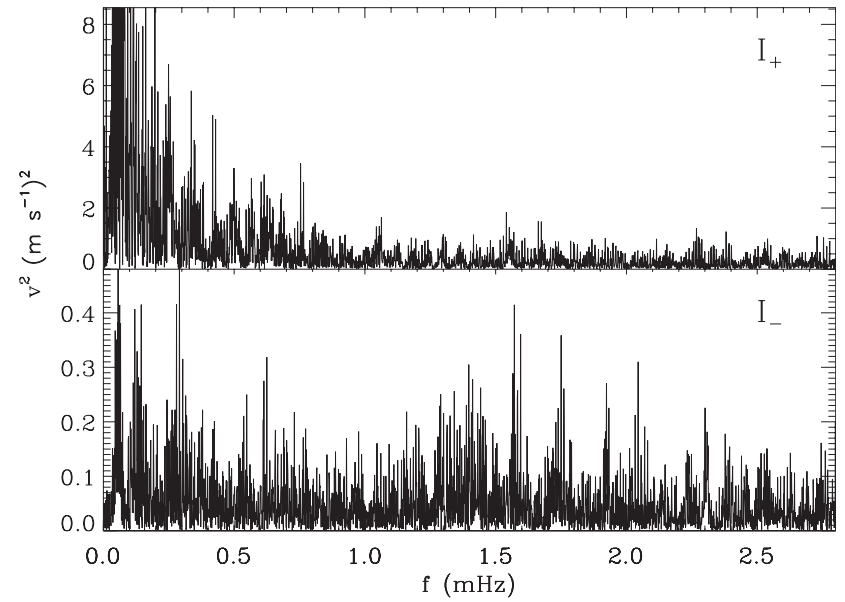

Fig. 4. Fourier spectrum of the time series $I_{+}$(upper curve) and $I_{-}$ (lower curve), with a signal translated in velocity. The spectrum of $I_{+}$, representative of the deformation of the mean line, does not show any excess power in the frequency range [1,2.2 $\mathrm{mHz}$ ], contrary to the one of $I_{-}$, very similar to Fig. 3 .

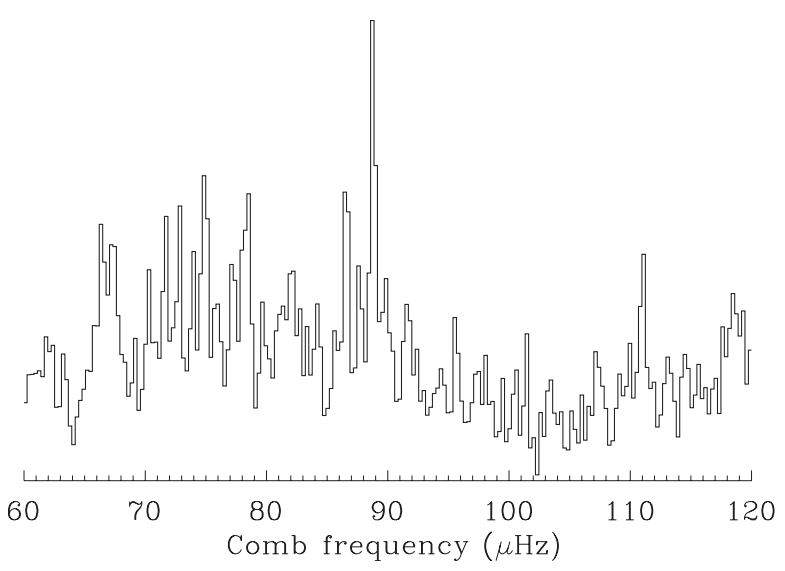

Fig. 5. Comb response of the spectrum of $I_{-}$between 1.2 and $2.2 \mathrm{mHz}$. The signal around $88.7 \mu \mathrm{Hz}$ is the signature of low degree solar-like pressure modes.

sensitive to the line broadening and distortion, while $I_{-}$is not, but sensitive to a line shift. This treatment is then able to extract the information of a pure Doppler signal, and confirms that the observed Fourier spectrum is not affected by distortions.

\section{The seismic signature}

The Fourier spectrum shows an excess power in the frequency range [1.2, $2.2 \mathrm{mHz}$, peaking at $1.7 \mathrm{mHz}$ near the predicted value of $1.3 \mathrm{mHz}$ derived from the scaling law Bedding \& Kjeldsen (2003). The comb analysis (Mosser et al. 1998) exhibits a clear signature around $\Delta v=88.7 \pm 0.4 \mu \mathrm{Hz}$ (Fig. 5), representative of the seismic large separation. We computed evolutionary models for HD 49933, using the CESAM code (Morel 1997). We considered the global parameters of HD 49933 $\left(6500 \mathrm{~K},[\mathrm{Fe} / \mathrm{H}]=-0.37, M_{\mathrm{V}}=3.39\right)$ and classical physical options for this kind of objects. Heavy element abundance 
$Z=7 \times 10^{-3}$ is deduced from $[\mathrm{Fe} / \mathrm{H}]$ and we adopted for helium $Y=0.255$ with $Y_{\text {primordial }}=0.240$ at $Z_{\text {primordial }}=0$, with $Z / X_{\odot}=0.0245$ and an enrichment galactic law $\mathrm{d} Y / \mathrm{d} Z=2$. The low metallicity induces a small mass for this range of temperature, so that the preferred mass is around $1.15 M_{\odot}$, with an age between 3.3 and 4.0 Gyr. For all computed models, the associated seismic large separation is found to vary between 75 and $88 \mu \mathrm{Hz}$. The $88.7 \mu \mathrm{Hz}$ spacing found in the data is thus consistent with this range of expected values, and is in favor of a young age.

In order to estimate the maximum oscillation amplitude, we have constructed synthetic time series, based on a theoretical low degree p-modes eigenfrequency pattern. The modes lifetimes are estimated from the eigenfrequency widths, between 1 and $4 \mu \mathrm{Hz}$ FWHM (Houdek et al. 1999). The maximum amplitudes are assumed to follow a Gaussian distribution in frequency. The synthetic power spectra are then calculated using the model of a stochastically excited, damped harmonic oscillator (Anderson et al. 1990). We assume that the spectrum is composed of this synthetic signal and a white noise. The maximum amplitude was then determined in order to obtain comparable energy per frequency bin in the synthetic and observed spectra. A Monte-Carlo approach finally shows that the best agreement is for a signal maximum amplitude about $0.4 \pm 0.1 \mathrm{~m} \mathrm{~s}^{-1} \mathrm{rms}$, with a Gaussian envelope centered at $1.7 \mathrm{mHz}$ and with a $1.0 \mathrm{mHz}$ FWHM. This estimate is not much sensitive to the assumed eigenfrequency widths: an uncertainty of $\pm 50 \%$ of their values adds an uncertainty smaller than $15 \%$. The agreement with the predicted scaling $\left(60 \pm 5 \mathrm{~cm} \mathrm{~s}^{-1} \mathrm{rms}\right)$ appears to be marginal.

\section{Conclusion}

We have been able to measure with HARPs the p-modes oscillation amplitude and the large separation of HD 49933. This confirms that ground-based asteroseismologic observations with HARPS provide excellent solar-like oscillations studies, even for a faint $\left(m_{v} \simeq 5.7\right) \mathrm{F}$ type star with a $v \sin i$ near $10 \mathrm{~km} \mathrm{~s}^{-1}$ and exhibiting activity. For a star such as HD 49933, ground-based spectrometric measurements are less efficient than for cooler types stars, with more and narrower lines. However, we have demonstrated that spectrometric measurements are the appropriate tools for disentangling the signatures of oscillations and activity. This problem is particularly important for intermediate mass stars, as most of the COROT targets, that contain information on the extension of the core and on the angular momentum transfer in the early phases of their lives. The question of contamination of the seismic signal by activity in the p-mode frequency range has been raised by Matthews (2004) as a possible cause of the non detection of oscillations on Procyon with MOST. Our observations allow an unambiguous distinction between the stellar activity and the seismic signal.

Acknowledgements. We thank the Genève group of HARPS for his precious help before and during the run. We thank Christophe Lovis for the follow up measurements conducted with CORALIE at the swiss 1.2-m telescope, Pierre Magain and Michael Gillon for modelling the stellar spectrum. We also thank the anonymous referee for his constructive comments.

\section{References}

Anderson, E. R., Duvall, T. L., \& Jefferies, S. M. 1990, ApJ, 364, 699 Baglin, A., Auvergne, M., Catala, C., et al. 2002, in Radial and nonradial pulsation as probes of stellar physics, ed. C. Aerts, T. R Bedding, \& J. Christensen-Dalsgaard, ASP Conf. Ser., 259, 626

Baranne, A., Queloz, D., Mayor, M., et al. 1996, A\&AS, 119, 373

Bedding, T. R., \& Kjeldsen, H. 2003, Publ. Astron. Soc. Austr., 20, 203

Bouchy, F., Pepe, F., \& Queloz, D. 2001, A\&A, 374, 733

Bouchy, F., \& Carrier, F. 2002, A\&A, 390, 205

Carrier, F., \& Bourban, G. 2003, A\&A, 406, L23

Connes, P. 1985, Ap\&SS, 110, 211

Donati, J. F., Semel, M., Carter, B. D., Rees, D. E., \& Cameron, A. C. 1997, MNRAS, 291, 658

Gray, D. 1992, The observation and analysis of stellar photospheres, 2nd ed., Cambridge Astrophysics Series (Cambridge University Press)

Houdek, G., Balmforth, N. J., Christensen-Dalsgaard, J., \& Gough, D. O. 1999, A\&A, 351, 582

Kurucz, R. L. 1979, ApJS, 40, 1

López-Santiago, J., Montes, D., Fernández-Figueroa, M. J., \& Ramzey, L. W. 2003, A\&A, 411, 489

Matthews, J., Kuschnig, R., Guenther, D. B., et al. 2004, Nature, 430, 51

Morel, P. 1997, A\&AS, 124, 597

Mosser, B., Maillard, J. P., Mékarnia, D., \& Gay, J. 1998, A\&A, 340, 457

Pepe, F., Mayor, M., Galland, F., et al. 2002, A\&A, 388, 632

Queloz, D., Mayor, M., Weber, L., et al. 2000, A\&A, 354, 99

Samadi, R., Georgobiani, D., Trampedach, R., et al. 2004, Excitation of solar-like oscillations accross the HR diagram, A\&A, submitted

Solano, E., Catala, C., Garrido, R., et al. 2004, Gaudi: preparatory archives for the COROT mission, AJ, in press http://sdc.laeff.esa.es/gaudi/ 